\title{
The Individual's Perception Space of the Digital Earth
}

\author{
Yuri Baturin $^{1}$ \\ 1 S.I. Vavilov Institute for the History of Science and Technology, Russian Academy of Sciences, \\ Baltiyskaya Str., 14, Moscow, 125315, Russia
}

\begin{abstract}
Just as the space of the real world is represented in the human brain (in its perceptual space) not as it really is, so the space of the Digital Earth is represented in the individual's space perception in a distorted way. The mechanism of forming an idea about an object in the perception space of an individual is represented. The structure of the Digital Earth space is discussed. The concept of "virtual-shift" is introduced. It is concluded that the perceptual spaces of the real world and the Digital Earth are turning into a single complex, which can help make the management of sophisticated objects, such as the economy of a particular country or the world economy, more adequate, but can also seriously disrupt it. Assumptions are made as to how this complex is represented in the space of perception of an individual and what are the consequences for the management of the country and international systems.
\end{abstract}

\section{Keywords}

Digital Earth, brain, sensation, cognitive perception, space of perception, virtual-shift

\section{Introduction}

Throughout history, people's perception of the space in which they live has changed, from a flat Earth to a view of our planet from space. Seeing fragments of the Earth has led to the notion of the 'cartographic image', which underpins the patterns of formation and perception of cartographic imagery. A cartographic image can be defined as a stable spatial representation formed as a result of the subject's accumulated experience while studying the map. As a result, a certain perception of the Earth as a whole has been formed. The interaction between the cartographic image and the perceiving subject generates information. Thus, cartography has been directly linked to information theory since its inception, to computer science, and finally to information technology. This link has not only survived the transition from cartography to neogeography, but has become crucial.

\section{Cognitive Perception of Space}

The Earth and the human being exist in three dimensional space. The visible picture is reflected on the light-sensitive retina and the reflected data goes to the brain, which uses the image on the retina to recreate an image of the outside world - also three-dimensional, but different from the real one. The process of transforming external space into perceptual space consists of stages described by different physics: the formation of the image of the object on the retina by optics, and the processing of the image by quantum mechanics. Thus, the external space undergoes essential transformations, so its geometry built by brain is not evident a priori. The image of space created in the human brain B.V. Rauschenbach called perception space. He showed that human perception space is not Euclidean, but Riemann space of variable curvature. In perception space, the rails in the distance converge to a point, whereas in real space they are always parallel.

So, the image on the retina (image of an object) is the input for visual perception. The retina has a layered, i.e. three-dimensional structure, which also consists of three-dimensional layers. It converts the

GraphiCon 2021: 31st International Conference on Computer Graphics and Vision, September 27-30, 2021, Nizhny Novgorod, Russia EMAIL: baturin@ihst.ru (Yu. Baturin)

ORCID: 0000-0003-1481-5369 (Yu. Baturin)

$\begin{array}{ll}\text { (c) (i) } & \text { (c) } 2021 \text { Copyright for this paper by its authors. } \\ \text { Use permitted under Creative Commons License Attribution } 4.0 \text { International (CC BY 4.0). } \\ \text { CEUR Workshop Proceedings (CEUR-WS.org) }\end{array}$ 
image of the object into electrical signals that are transmitted via the optic nerve to the brain. The result of the image conversion depends on the spatial distribution of the photoreceptor sticks and cone cells in the last layer of the retina and how these cells are linked to the ganglion cells. Cones are responsible for spatial sensitivity. Signals from the photoreceptors eventually reach the ganglion cells, whose axons run in the optic nerve and carry information to the brain. The optic nerve fibres coming from each retina split into two bundles at the optic crossroads and end in the thalamic cell layer, in the lateral geniculate nucleus. Axons of geniculate nucleus neurons, in turn, pass through the optic radiation (Gratiolet bundle) into the visual cortex of both cerebral hemispheres [2].

Visual information reaches the brain via the optic nerve in two pathways. One, from the retina, passes through a midbrain structure called the superior upper two bumps (superior colliculus), that notifies other brain structures of what is being seen, and then projects through the pulvinar (thalami) into the parietal lobes. This pathway has to do with the spatial aspects of vision: it makes it possible to understand where an object is located, but not what it represents. The second pathway allows the analysis and recognition of complex visual scenes and objects. It is the pathway from the retina to the primary visual cortex (usually labelled V1), the oldest and simplest of the cortical areas, but the most studied. The information is then split into two streams: (the "how" stream) and (the "what" stream). The function of the 'how' stream is somewhat the same as that of the first pathway, but it provides much more complex aspects of spatial vision - determining the overall spatial arrangement of a visual scene, not just the location of an object. The 'how' stream projects into the parietal lobe and has close connections with the motor system [3].

At each stage of transfer of visual information it is analysed, processed and ... then already other, modified information is transferred. So, for example, if information of illumination level is received from photoreceptors, ganglion cells transfer information about difference in illumination levels within an image of object.

Admiration of the amazing system, which allows us perceiving the world around us, cannot be conveyed by strictly scientific language. We will therefore conclude this subsection with the poetic words of Charles Scott Sherrington, winner of the 1932 Nobel Prize in Physiology or Medicine:

"...Our eye, while we look, throughout the day sends out constant rhythmic streams of tiny, detached, ephemeral electrical potentials into the dense forest of cells and fibres of our brain. This pulsating and flowing company of electrical discharges through the spongy structure of the brain bears no visible resemblance to the spatial or temporal structure of the surrounding world; it only remotely resembles that tiny two-dimensional and, additionally, upside-down picture of the surrounding world that appears in the eye at the beginning of the nerve fibres, before the storm of electrical discharges. And this storm of discharges is arranged in such a way that it affects the functioning of brain cells of all types. Electrical discharges themselves do not carry even the slightest bit of visual information, having nothing to do, for example, with such concepts as "distance", "right-left", nor "vertical" or "horizontal", nor with "colour", "brightness" or "shadow", nor with "roundness" or "squareness", nor with "contour", "transparency" or "opacity", nor with the notions of "far" or "near", nothing with any visual information at all - nevertheless allow us, as if by magic, to imagine it all" [4].

But the mechanism of perceptual space formation and the process of perception is even more complex than the picture described before such a beautiful quote.

\section{Sensory Feeling of Space}

The perception of space begins to form in the individual at birth, scientifically speaking, with the beginning of his interaction with the environment, and is confirmed by experience then, but also changes in the course of changes in the external environment. By nature, man is endowed with perceptive organs - not only the brain, which carries out the second phase of perception, but also the receptors. Even in his or her unconscious actions he or she is guided by the reactions of sensory receptors (sensors). The reactions of the receptors (sensation) are the result of their interaction with the environment and depend on the properties of the environment. The recording of environmental influences provides information that is statistically correlated with trends in environmental conditions. Thus, individuals and their sensory systems are causally related to the environment, which is functionally explained by the value of the information that the environment conveys to individuals' sensory systems. Differences in receptor 
(sensor) responses depend on differences in environmental properties. That is, the individual has an innate ability to detect differences in properties, which allows him or her to distinguish between himself or herself and the environment. In addition to the individual's capacity to detect the presence of boundaries, the individual also has the capacity to detect the spatio-temporal characteristics of the environment. This is how the individual begins to represent space, through sensation (registration of information by receptors). Information registration by receptors is the sensory capacity for objectified representation of an object [5].

Sensory feeling and cognitive perception complement each other. It is cognitive perception that firstly forms the perceptual space in the brain. Consciousness begins to interpret sensory data as well, objectifying them, which entails the representation of the object in the perceptual space.

Accordingly we will distinguish systems and states of sensory information registration from systems and states of the subject's cognitive perception. Both types of systems and states are functional with the ultimate goal of constructing the subject's representation of the object. Functional information transfer ("information registration"), clarifies the understanding of the subject's sensory systems, and complements the classical notion of "information transfer".

The environment in which an individual is formed is complex. It can be divided into social, natural and technical components. In what follows, we will be particularly interested in the IT part of the technical component of the environment. Particularly significant changes are taking place today due to the rapid development of the IT-environment.

The highly complex system of subtle perception mechanisms described above must inevitably, at least in part, change its mode of functioning under the influence of such rather crude tools as information technology (even before the emergence of neuro-information technology; let us imagine that it does not exist and will not be described in this article).

\section{Digital Earth}

The emergence of the Digital Earth [6] means the emergence of a new perception characteristic of a significant part of humanity that has grown up in an environment of information technology. And the perceptual space by the subject of the Digital Earth is not at all a variation of the perceptual space of the Earth, its fragments and cartographic images. The changes are so cardinal that it will take time to recognise, study and understand the changes that have occurred, and to be able to prevent or minimise their negative effects.

A Digital Earth is a set of digitized, linked and represented objects, in accordance with the principles of neogeography. The backbone of the Digital Earth is the Internet, whose topology (as well as other computer networks) includes specfic neogeographic objects and users.

Indeed, the Internet space is not only a complex technical object, but also a community of individuals entering into various connections and various relationships with each other regarding the information circulating in the global infrastructure of Digital Earth. But how is the technical network of the Internet connected to the community (network) of persons where, in fact, much of life on Digital Earth unfolds? So, there is an Internet space as a complex technical object, as a network of technical devices. A person appears (or can appear) at every node of technical network. This means that we can speak of a kind of social network, or rather a multitude of realizations of a common social network, corresponding to the technical structure of the Internet. Nodes are human inner worlds, but the meaning of the Internet is hidden not in the nodes, but in the connections between them, i.e. in the relations between the members of the emerged community. Through established connections, new content is induced (transferred) in other nodes of the network. In fact, over the technical part of the network defined by physical links and connection points (nodes), a second - field (induced, transferred) network of connections emerges, describing the circulation of ideas, discussions, finally, public opinion, which defines the community. But how do these two networks create unity? As long as a technical network (Internet) is not induced (not excited by human informational influence), it is just a physical scheme. The induced (excited) network is the human essence of the Internet. Thus, the nodes are linked together not only by physical links, but also by "force lines" of the induced field. And once the relationship between the "nodes" has emerged, it requires social regulation. In this sense, Digital Earth as a space "stretched" on the Internet becomes not only a technical, but also a social phenomenon [7]. 
Digital Earth is evolving rapidly, as is its configuration space, the "receptacle" of the phenomenon itself, a well-visualized network (many are familiar with diagrammatic pictures of this kind) stretched over many reference points located all over the planet and even in near-Earth (so far) outer space. Mankind cautiously enters new nooks of Digital Earth and begins to explore them. But the open subspaces of the Digital Earth pose many practical questions for scientists, which are not easy to solve. But they must at least be formulated and posed.

\section{Perceptions of the Real and the Virtual}

Global digitalization and information technology have given rise to many virtual worlds and the objects within them. The notion of virtuality comes from the Latin virtualis, an object or state that can arise here and now under certain conditions. They can arise in the mind, but they can be created in the digital world.

A reference to Latin roots tells us that virtual objects were known as far back as Ancient Rome. Indeed, Roman jurists used such a construct as "legal fiction". It consists of the assumption that a nonexistent fact is recognised as existing or vice versa, which had a certain legal value. The idea of fiction was not originally associated with legal procedures in any way. Roman lawyers took it from the Stoics, who used it to construct a clear and logical system of meanings. With the help of fictions (i.e. of what does not exist, but can be) a consistent "semantic space" was being built. It was already then, in modern terms, partly virtual.

Until the end of the twentieth century, virtuality was understood as arising in the mind. That changed as we entered the digital age. New ways of virtualization have emerged. And the relationship between real and virtual spaces began to shift in favour of virtual. There was a "virtual-shift" effect - the boundary between the real world and the virtual one began to move [8].

Digital Earth is a virtual object in virtual space. In a narrower sense, it is a specific virtual space. It is a "semantic fiction" - something that does not exist, but could be. How is this virtual object perceived by man and humanity?

The absence of sensory information in the virtual environment is analogous to observation from the outside (contemplation) but not to dealing directly with the object. Perception is sensation interpreted by consciousness. Pure contemplation contains nothing of sensation. A considerable amount of information about the environment is lost, i.e. only the "appearance" of the object is created. Therefore, in computer games, players make up information that is not sensed. Some of the information that reaches the brain is false. When perceiving visual images in virtual worlds, the brain receives some sensory information - from the joystick, keyboard, touch screen, etc. This information can be erroneously interpreted as signs of reality, i.e. will mislead the mind. Some of the information intended for the brain is just an imitation of some process, for example with gloves that allow one to feel the volume and texture of virtual objects, or with suits in which the user feels the rain drops and gusts of wind "falling" on them. In virtual reality, it is even possible to create sensations that are not realizable in the real physical world and fictional environments.

A person, through sensory feeling and visual information systems, makes hypotheses about what they are dealing with based on their previous experiences in the virtual world - and these hypotheses are mostly wrong for the real world. "Memory of perception" - both real and virtual - has a way of losing detail. But the lost element of virtual memory is not necessarily replaced by some real-world memory match. "Memory of perception" creates inertia in the perceptual space and promotes the mixing of the virtual and the real in consciousness. Perception changes our condition. Virtual "shifted" perception, subject to loss of memory elements, becomes potentially dangerous to the real world.

The child perceives the fairy tale as reality. Not only children, but also young people who have spent most of their lives in virtual spaces, not only having had fun, but also studying and working in them, see them as a form of travelling abroad, studying at a foreign university or working for a foreign company. The age boundary between the perception of the real and virtual worlds is visibly shifting to the adult part of a person's life. People who have grown up in and been brought up in the digital virtual world begin to manage us too (what kind of management should be in order to evoke adequate responses in the real world is a separate question). A "governance gap" is emerging. 


\section{Properties of Digital Earth Space and Digital Earth Perception Space}

Digital Earth space, as perceived by man, is an informational virtual space, surrounding real Earth space, which is gradually contracting. And it is not a spherical compression uniform over the entire sphere's surface, but a certain hypersphere, i.e. a sphere with deformations - hummocks (convexities) appearing where new virtual images are created faster, and caverns (voids) where they fail.

Thus, the Digital Earth is an allocentric (Greek allos - other, kentron - tip of a compass) information system, i.e. something external that is the basis for perception of the observable. This information system relates to a grid of metric spatial relations and functions to enable us to study any fragment of the terrain. The out-of-scale nature of the Digital Earth [9] generates self-similarity (leading to a scaling effect) [10], and a self-similar object must be fractal. Consequently, perception space reflecting it turns out to be fractal too, i.e. the dimensionality of Digital Earth information space and its perception space in general case will be fractional.

The grid of metric spatial relations sets a digital image of space. In this mathematical structure, the process of grid cell reduction could go on indefinitely deep, but for practical reasons it is reduced only to the pixel level which guarantees sufficient accuracy of the Digital Earth representation. In fact, the pixels of digital space (Digital Earth in particular) correspond to "quanta" of perceptual space, making it discrete (not continuous), quantized. The space of the Digital Earth is discrete in a particular case, but generally speaking, discontinuous.

The Digital Earth can be viewed through the complex structure of configurational Internet space, its stratifications (Tor Browser, Dark Net), through the deep floors of Internet space - various kinds of online universes (worlds), etc. This means that the Digital Earth is a heterogeneous space, unlike the real space in which we live. The inhomogeneities of Digital Earth will make its perceptual space inhomogeneous.

Closedness, discreteness, inhomogeneity are the main properties of the hollow, fractional dimensional perception space of the Digital Earth. It must be admitted that mankind has never encountered such complex objects before.

Thus, the perception space of the Digital Earth is non-Euclidean. It is not the space of hyperbolic or elliptic geometry (Lobachevsky space or Riemann space). It is hollow, inhomogeneous, asymmetrical, with ridges and cavities, folds and kinks, a space of fractional dimension, where causal links are far from obvious.

The real world, or rather the space of its perception, is "hidden" inside the subject's space of perception of the "Digital Earth", and the layer that "filters" perception is getting thicker and separates us from the perception of reality more and more. The perceptual spaces of the real world and the Digital Earth are turning into a single complex, which can help make the management of sophisticated objects, such as the economy of a particular country or the world economy, more adequate, but can also seriously disrupt it.

Without an understanding of this problem and a conscious response to it, we run the risk of our complex real world being described in a consistent and strictly logical manner by predominantly virtual fictions with solutions that are simple, understandable, but not satisfactory to us in terms of results.

\section{Conclusion}

Management with "virtual shifts", produced in the real world, by the world economy, international relations and internal management of the economy in countries will seem to older generations to be plunged into a world of absurdity, while younger generations will regard it as perfectly normal. There is a theorem in physics called R. Gerock's Theorem, from which it follows: if there is a change in topology in any physical process, it will initially be perceived by an external observer (in terms of the old topology) as an unexpected abrupt change in the law of causality [11]. If we try to generalize this theorem to social processes, we may suppose that soon we will have to face more and more often illogical developments, seemingly broken cause-and-effect relations, "absurd" decisions, indicating deformation and, perhaps, approaching sharp break of all life space.

When the change of generations takes place, the world will simply become different: reality will become less, virtuality will be recognized as a great convenience, people will acquire virtual attributes 
and become the humanity of the Digital Earth with an adequate perception of it. Real space and planet Earth itself will probably become studied as examples of a distorted and primitive virtual perception of Digital Earth.

Difficulties are inherent in the transitional period, but it is not so short. And it is under transitional conditions that development can take unexpected paths.

\section{References}

[1] B.V. Raushenbakh, Prostranstvennyye postroyeniya v zhivopisi. Ocherk osnovnykh metodov, Nauka, Moscow, 1980. (In Russian)

[2] I.P. Hermann, Physics of the Human Body, Springer-Verlag Berlin Heidelberg, 2007.

[3] V.S. Ramachandran, The Tell-Tale Brain. Unlocking the Mystery of Human Nature, Windmill Books, London, 2011, pp. 62-63.

[4] J. G. Nicholls, A. Robert Martin, B. G. Wallace, P. A. Fuchs, Ot neyrona k mozgu. M., LIBROCOM, 2012, p. 440. (In Russian. Original title: From Neuron to Brain).

[5] Yu. M. Baturin, Limits of "the Self", Boundaries of Personal Space and IT, in: Proceedings of the 2021 International Conference Engineering Technologies and Computer Science EnT 2021. Moscow, Russian Federation 18-19 August 2021. - Los Alamitos, 2021, p. 30-31. doi: 10.1109/EnT52731.2021.00012.

[6] A. Annoni, M. Goodchild, H. Guo (eds), Manual of Digital Earth, Springer, 2019, doi: 10.1007/978-981-32-9915-3.

[7] Yu. Baturin, Auto-Audit of Digital Earth and Beyond. Geocontext 8 (1) (2020) 17-21. URL: https://geo-context.org/index.php/geocontext/article/view/50 (In Russian)

[8] Yu.M.Baturin, S.V. Polubinskaya, What makes virtual crimes to be real, Proceedings of the Institute of State and Law of the RAS 13(2) (2018) 30-31. (In Russian)

[9] E.N. Eremchenko, V.S. Tikunov, A.A. Zakharova, Digital Earth and "Digital" initiatives of Modern Era, in: GraphiCon 2018: Proceedings of the 28th International Conference on Computer Graphics and Machine Vision, Tomsk, 2018. pp. 304-306. (In Russian)

[10] B.B. Mandelbrot, The Fractal Geometry of Nature. W.H. Freeman and Company, N.Y., 1977.

[11] R. Gerock, Topology in general relativity, Journal of mathematical physics 8 (1967) 782. 